\title{
Evaluación de un Plan de Desarrollo Docente Universitario basado en la Experiencia: el Modelo de Aprendizaje 70:20:10 en la Universidad Europea de Madrid
}

\author{
María A. Ruiz-Rosillo , María D. Vivas-Urias, Eva Jiménez-García y Sara Redondo-Duarte \\ Vicerrectorado de apoyo a la Docencia y a la Investigación, Unidad de Innovación Educativa y Desarrollo Docente, \\ Universidad Europea de Madrid, C. Tajo s/n, Villaviciosa de Odón, 28670, Madrid, España. \\ (e-mail: mariaauxiliadora.ruiz@universidadeuropea.es; mariadolores.vivas@universidadeuropea.es; \\ eva.jimenez@universidadeuropea.es; sara.redondo@universidadeuropea.es)
}

* Autor a quien debe ser dirigida la correspondencia

Recibido Oct. 16, 2018; Aceptado Dic. 19, 2018; Versión final Feb. 21, 2019, Publicado Ago. 2019

\begin{abstract}
Resumen
Se ha investigado la participación y valoración de los docentes sobre el nuevo Plan de Desarrollo Docente, implantado por La Universidad Europea de Madrid, una universidad privada en España. El plan está basado en el modelo de aprendizaje 70:20:10, e introduce nuevas oportunidades de aprendizaje con el fin de impulsar la mejora e innovación en la formación continua del profesorado. Se estudia también la relación existente entre el conocimiento de este modelo y el nivel de satisfacción con el mismo. El enfoque metodológico es de carácter mixto y los resultados nos permiten concluir que el nuevo Plan es percibido positivamente por el claustro. Se demuestra una relación positiva y significativa entre las valoraciones sobre el Plan y la satisfacción con el mismo. Las principales recomendaciones instan a continuar trabajando en la consolidación del nuevo Plan, dando un mayor peso al aprendizaje colaborativo y a la experimentación como vías prioritarias de formación docente.
\end{abstract}

Palabras clave: desarrollo docente; oportunidades de aprendizaje; modalidad formativa; formación continua; educación superior

\section{Assessment of an Experienced-Based University Faculty Development Plan: The 70:20:10 Learning Model at the European University of Madrid}

\begin{abstract}
This paper presents an investigation about teachers' participation and their evaluation regarding to the new Faculty Development Plan in 2016 implemented by the European University of Madrid, a private university in Spain. The Plan is based on the 70:20:10 learning model and incorporates new learning opportunities in order to promote improvement and innovation within the continuing faculty training. Also, the correlation between the knowledge about this model and the satisfaction level with the plan is studied. The results demonstrate a positive and significant relationship between the evaluations on the Plan and the satisfaction with it. The main recommendations urge to continue working on the consolidation of the new Plan, giving greater weight to collaborative learning and experimentation as priority development paths.
\end{abstract}

Keywords: faculty development; learning opportunities; training modality; continuous training; higher education 


\section{INTRODUCCIÓN}

La Universidad Europea de Madrid, en adelante UEM, es un centro educativo español constituido oficialmente como universidad privada en 1995 que ofrece formación universitaria de grado, postgrado, doctorado y formación profesional de grado superior. Su misión es la de proporcionar a sus alumnos una educación integral, formando líderes y profesionales preparados para dar respuesta a las necesidades de un mundo global, aportando valor en sus profesiones y contribuyendo al progreso social desde un espíritu emprendedor y de compromiso ético. El modelo educativo de la UEM se enmarca dentro de un enfoque humanista de la educación, centrado en el desarrollo de la persona, en su autorrealización y en la educación de las emociones. El estudiante aprende siendo y haciendo, responsabilizándose de sus elecciones y consecuencias, desarrollando las competencias, conocimientos y valores que demanda la sociedad actual. La aproximación a la docencia en nuestra institución supone necesariamente trascender a la mera adquisición del conocimiento para abordar el desarrollo de un aprendizaje integral e integrado, propiciando que los estudiantes adopten un papel activo en su propio aprendizaje. Siendo este es uno de los principales retos del Espacio Europeo de Educación Superior (Garmendia et al., 2014). En este contexto, el Plan de Desarrollo Docente en nuestra Universidad (PDD en adelante) pretende apoyar al claustro en el desarrollo y la mejora continua de las competencias necesarias para desempeñar sus principales funciones (Mas y Olmos, 2016): docente, investigadora y gestora. Son numerosos los estudios que se han realizado para identificar y validar el perfil competencial del docente universitario (Villarroel y Bruna, 2017), sin embargo, no existe un consenso al respecto, por lo que se consideran los tres niveles indicados por la Comisión Europea (Tejada, 2013): 1) Competencias profesionales, relacionadas con la experiencia laboral y las destrezas técnicas profesionales; 2) Competencias pedagógicas y sociales, que facilitan los procesos didácticos, el trabajo con los jóvenes, la integración de las funciones formativas, la mentorización, el aprendizaje colaborativo y la trasferencia efectiva de conocimiento; y 3) Competencias de gestión, coordinación con agencias formativas colaboradoras y supervisión de prácticas.

En este contexto, los programas de formación continua del profesorado universitario se han centrado predominantemente en el desarrollo de la competencia pedagógica (Madinabeitia y Lobato, 2015), ya que como indica Zabalza (2009:78), "formarse pedagógicamente es el compromiso que asumimos con nuestros estudiantes y con nosotros mismos para activar todos los recursos que estén en nuestra mano para mejorar su formación". Este es el caso del programa de formación continua del profesorado que nuestra institución había implementado hasta 2015, momento en el que se evaluó su efectividad y, como resultado, se detectaron tres aspectos fundamentales de mejora: 1) promover mayor conexión entre la formación docente y su ámbito profesional; 2) responder a distintas necesidades y maneras de aprender, de un modo más atractivo y diverso; y 3) involucrar a los mejores docentes para promover de manera más efectiva el intercambio de buenas prácticas. Por lo tanto, la conclusión de este análisis puso de manifiesto la necesidad de evolucionar y transformar este programa formativo.

\section{PLAN DE DESARROLLO DOCENTE EN LA UNIVERSIDAD EUROPEA DE MADRID}

Como paso previo al rediseño del programa formativo en la Universidad, se revisaron los programas de otras universidades, consideradas como referentes en educación superior por su posicionamiento en rankings internacionales de prestigio, pues la evolución de los programas de desarrollo docente no ha ocurrido simultáneamente ni de la misma manera en todos los países (Madinabeitia y Lobato, 2015; Martínez et al., 2018). Se observó que los programas de las universidades estudiadas se centraban, fundamentalmente, en el desarrollo de competencias y habilidades para la mejora de la práctica en el aula y del propio desarrollo profesional (Ion y Cano, 2012; Boud y Brew, 2013). Además, utilizaban plataformas y centros de tecnología educativa con recursos de apoyo a la actividad académica, y promovían y reconocían la excelencia de la enseñanza.

En este estudio destacaron los casos de Eaton y de Princeton University (2015), por su adopción del modelo de aprendizaje y desarrollo basado en la experiencia 70:20:10 (McCall y McCauley, 2014). Este modelo es el resultado de las investigaciones llevadas a cabo por Lombardo y Eichinger (1996) en el Center of Creative Leadership acerca de cómo los ejecutivos aprenden, crecen y cambian a lo largo de sus carreras. Las conclusiones sostienen que la mayor parte del aprendizaje en una organización es informal y ocurre naturalmente en el lugar de trabajo (Cross, 2007; Webster-Wright, 2009), proviniendo aproximadamente el $70 \%$ de la experiencia, bien en el trabajo o en la vida real; el $20 \%$ de la retroalimentación, de la observación y el trabajo con los demás; y el 10\% de cursos tradicionales (Oliver et al., 2009; Vallejo y Wehn, 2015). Según el estudio de Jennings et al. (2016), el 47\% de los responsables de aprendizaje y desarrollo implementa modelos en los que el aprendizaje se apoya en el flujo de trabajo, y reportan más beneficios que aquellos que no lo hacen. Al margen del tipo de modelo de aprendizaje basado en la experiencia implementado, más de un $50 \%$ de estos responsables indican que sus organizaciones son más ágiles y productivas, y más de un $70 \%$ reportan que sus procesos son más eficientes. Las críticas hacia este modelo 
señalan el riesgo de exagerar el rol de la experiencia en el desarrollo del talento y subestimar el valor de aprender de los demás, así como la falta de orientación detallada y específica sobre las lecciones aprendidas de cada experiencia (Garavan et al., 2012). En base a estos resultados, y atendiendo a la premisa de adoptar un modelo más ligado a la realidad del docente, que parta de sus experiencias y le permita adquirir protagonismo en su proceso de desarrollo profesional (Desimone, 2009; Boud y Brew, 2013; De Rijdt et al. 2013; Santos y Sarceda, 2017), se decidió utilizar el modelo 70:20:10 como base del nuevo programa de formación continua. Durante este proceso de diseño, se llevó a cabo la revisión de las áreas de desarrollo, los tipos de modalidades de aprendizaje y actividades formativas. Como resultado, en el año 2016 se implanta el nuevo Plan de Desarrollo Docente, estructurado en dos ejes: 1) Áreas de Desarrollo y 2) Modalidades de aprendizaje y tipos de actividades formativas.

\section{Áreas de desarrollo}

A diferencia del programa de formación anterior, el nuevo PDD comprende cuatro áreas de desarrollo que se alinean y pretenden cubrir las principales funciones del profesor universitario (Tejada, 2013; Mas y Olmos, 2016): 1) la pedagógica, vinculada al Modelo Académico de la Universidad y a la mejora docente; 2) la técnica, enfocada hacia la adquisición de competencias y habilidades específicas necesarias para el desempeño de labor del docente; 3) la gestión, vinculada al desarrollo de competencias y habilidades necesarias para el desempeño de determinadas funciones en la Universidad, como la coordinación de asignaturas o titulaciones, la coordinación académica, etc.; y 4) la investigación, centrada en el fomento de la labor investigadora mediante la participación en congresos científicos, la realización de publicaciones, y actualización de conocimientos y habilidades.

\section{Modalidades de aprendizaje y tipos de actividades formativas}

Un modelo de desarrollo docente basado en la experiencia supone un reto a la hora reconocer y contabilizar como horas de formación aquellas provenientes del aprendizaje informal. Para facilitar esta tarea se definen tres modalidades de aprendizaje que incluyen un catálogo de acciones formativas asociadas a cada una de ellas. De acuerdo con el modelo 70:20:10, en el PDD se establece que, en términos generales, el $70 \%$ de las horas de formación del docente han de corresponder con el autoaprendizaje en el trabajo, modalidad que se denomina Experimentar; el 20\% deben provenir del aprendizaje entre pares, modalidad que se denomina Compartir; y solo el 10\% debe emplearse en cursos tradicionales, de la modalidad Descubrir.

Por lo tanto, en la modalidad de Experimentar, los docentes aprenden haciendo a través del desempeño de alguna de sus funciones: docente, investigadora o gestora. En este contexto, la exposición de los docentes a oportunidades para experimentar diferentes situaciones organizativas, culturales, interculturales y profesionales, se destaca como una dimensión importante del desarrollo, pues implica una oportunidad para trabajar en diferentes contextos y situaciones (Garavan et al., 2012). Por ello, con el objetivo de contabilizar como formación las horas de aprendizaje experiencial realizadas, se reconocen como actividades formativas, entre otras: la transferencia de conocimientos y habilidades adquiridas en otros contextos; la aplicación de nuevas metodologías y/o tecnologías docentes; la labor investigadora (realización de publicaciones en revistas de impacto o participación en jornadas y congresos como conferenciante o comunicador); o la realización de estancias en una empresa y estancias académicas internacionales, siendo la movilidad un aspecto fundamental de la formación continua (Tejada, 2013). En la modalidad Compartir, los docentes aprenden colaborando a través de la observación formativa, actividad que tiene el objetivo de mejorar la práctica docente aprendiendo de la forma en la que otros enseñan (Martínez et al., 2018); la mentoría y la realización de proyectos de investigación-acción (Garmendia et. al, 2014); así como la participación en comunidades de práctica, en seminarios y en redes sociales. Por último, en la modalidad Descubrir, los docentes aprenden de la manera más tradicional, a través de cursos y programas formativos específicos con distintos niveles de dominio; sesiones plenarias impartidas por expertos; así como talleres prácticos enfocados a desarrollar conocimientos y habilidades sobre un tema específico.

Este programa constituye la oferta de formación continua para el profesorado de la institución y tiene un carácter obligatorio para los docentes a tiempo completo, que deben realizar 60 horas de formación. Por lo general, al inicio del año natural, los docentes definen su plan de desarrollo individual junto con su director de departamento siguiendo unas pautas básicas: 1) establecer entre uno y tres objetivos de desarrollo medibles, observables, temporales y alcanzables; 2) establecer para cada objetivo tipos de actividades formativas de acuerdo con el modelo 70:20:10. De manera formal y sistemática, a mitad de año se realiza una reunión intermedia de seguimiento entre docente y su responsable, con el fin de ajustar los indicadores de consecución y recopilar las evidencias para su posterior validación. Este proceso culmina con la evaluación al final de año, momento en el que se contabilizan y valoran las actividades de aprendizaje realizadas. De este modo, el Plan pretende capacitar a los profesores en la planificación y diseño actividades formativas que permitan a los estudiantes adquirir los resultados de aprendizaje previstos; la 
implementación de métodos de aprendizaje activos en el aula; la organización del proceso de aprendizaje en torno a problemas reales; la creación de un clima de investigación en el aula; y la promoción de la innovación educativa para la mejora continua.

Una vez conocido el diseño y la estructura del nuevo Plan, se ha propuesto para esta investigación un doble objetivo. Por un lado, se pretende conocer la percepción que tienen los docentes sobre él, para revelar los aspectos mejor evaluados y los que requieren de una mejora. Por otro lado, y con la intención de demostrar la importancia de diseñar y planificar adecuadamente un programa de desarrollo docente de este tipo, se pretende identificar la posible relación entre las variables sobre el conocimiento y estructura del PDD y el nivel de satisfacción de los docentes con el mismo.

\section{METODOLOGÍA}

El enfoque metodológico utilizado es de carácter mixto y engloba dos fases, una cuantitativa y otra cualitativa, cuya combinación resalta la solidez de las diferentes aproximaciones (Bryman, 2006). Para la revisión y valoración del PDD se utiliza la investigación evaluativa, con el fin de identificar fortalezas e impulsar mejoras en años sucesivos. En concreto, las variables que se analizan están relacionadas con el conocimiento que tienen los docentes respecto al Plan, la oferta formativa, la modalidad de aprendizaje, la contribución del aprendizaje en la mejora de la práctica docente y el nivel de satisfacción con el PDD.

En lo que respecta a los métodos cuantitativos, se emplea un diseño no experimental para analizar los resultados de la encuesta de Evaluación de la Actividad Docente, dirigida a los profesores de la Universidad, con el objetivo de recoger información cuantitativa sobre diferentes aspectos relativos a la docencia universitaria que permitan identificar fortalezas e impulsar mejoras en aquellas áreas donde sea necesaria. Consiste en un cuestionario formado por 38 preguntas cerradas, en las que se ha empleado una escala de actitud tipo Likert de cinco puntos, de entre las que se han seleccionado cinco preguntas correspondientes a aspectos relacionados con el Plan de Desarrollo del Docente: Pregunta 1. Conocimiento del PDD; Pregunta 2. Adecuación de la oferta formativa a tu desarrollo; Pregunta 3. Las modalidades de aprendizaje son adecuadas para tu desarrollo; Pregunta 4. Contribución de lo aprendido a la mejora de la práctica docente; Pregunta 5. Nivel de satisfacción con el Plan. La población objeto de estudio corresponde al conjunto de 1226 docentes de la Universidad Europea de Madrid, de los que 640 participan en actividades del Plan de Desarrollo y 464 docentes en la encuesta de Evaluación de la Actividad Docente. Se trata por tanto un diseño muestral por conveniencia, que según Casal y Mateu (2003) se realiza a través de métodos no aleatorios y la representatividad la determina el investigador. En ambos casos, el tamaño de la muestra es significativo, ya que atendiendo al nivel de confianza del $95 \%$ y un margen de error del $4 \%$, se exige como mínimo una muestra de 404 sujetos.

Las técnicas cualitativas empleadas consistieron en un grupo focal, ya que estos permiten obtener una mejor compresión de los sentimientos y creencias de los participantes, mediante la compartición de percepciones y puntos de vista acerca de un tema, idea o concepto (Krueger y Casey, 2009). Se seleccionaron ocho profesores de la Universidad utilizando un muestreo no probabilístico intencionado, en base a los siguientes criterios: tener al menos el $50 \%$ de los objetivos de su Plan individual superados; tener experiencia docente; y representatividad de las diferentes facultades. De este modo, se contó, tanto con perfiles docentes senior, con más de cinco años de experiencia en la Universidad, como con perfiles docentes junior, con una antigüedad de entre uno y dos años, pertenecientes a diferentes áreas de conocimiento. En el grupo focal los profesores compartieron información acerca de su experiencia con el PDD en relación con distintos aspectos, tales como: las modalidades de desarrollo, la oferta de acciones formativas, o la flexibilidad del Plan para el reconocimiento de acciones para el desarrollo.

\section{ANÁLISIS Y DISCUSIÓN DE RESULTADOS}

El análisis y discusión de resultados se presenta en cuatro subsecciones: los resultados cuantitativos del estudio, las medidas discrecionales, las medidas simétricas de concordancia y los resultados cualitativos analizados.

\section{Resultados cuantitativos}

Tras la implementación del nuevo PDD basado en el modelo 70:20:10, los datos globales de participación de los profesores en el año 2016 muestran que el 62,4\% de las horas dedicadas por los profesores a su Plan individual estuvieron vinculadas a la modalidad Descubrir, mientras que el $29,3 \%$ lo estuvieron con la modalidad Experimentar, y el 8,4\% con la modalidad Compartir. Con respecto a la distribución de las horas de formación contabilizadas como acciones formativas en cada modalidad de aprendizaje, en la modalidad Experimentar predominaron las horas empleadas en la participación en jornadas y congresos (figura 1). 


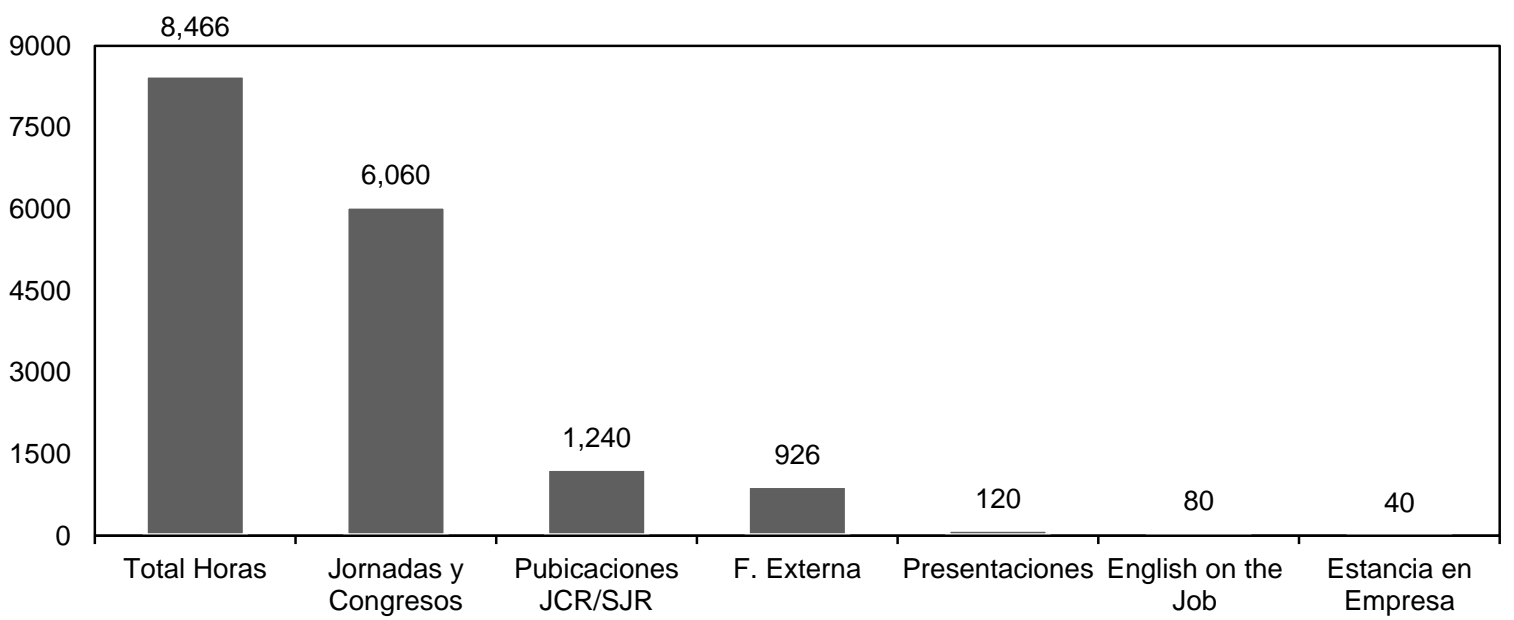

Fig. 1: Distribución del número de horas de formación en la modalidad de Experimentar

En la modalidad Compartir (figura 2) existe una mayor fragmentación, siendo los proyectos académicos $(46,3 \%)$, proyectos de investigación-acción $(23,6 \%)$ y la mentoría $(19 \%)$ las actividades a las que mayor número de horas han dedicado los docentes.

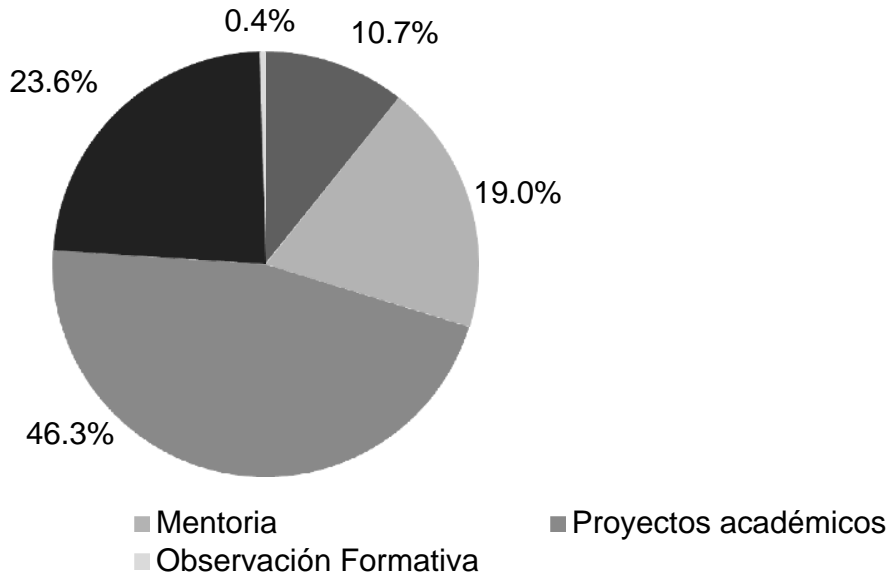

- Acompañamiento

- Proyectos $\mathrm{I}+\mathrm{A}$

$$
\text { Observación Formativa }
$$

Fig. 2: Distribución del número de horas de formación en la modalidad Compartir

Como resultados de la encuesta de Evaluación de la Actividad Docente (tabla 1) se destaca la distribución de las respuestas de los docentes y la media de su valoración con respecto al conocimiento del PDD, la oferta formativa, las modalidades de aprendizaje, la contribución del aprendizaje en la mejora de la práctica docente y el nivel de satisfacción con el Plan. Los mayores porcentajes se encuentran en la categoría Alto y las medias en todos los casos superan los 3 sobre 5 puntos.

Tabla 1: Valoraciones del Plan de Desarrollo Docente

\begin{tabular}{|l|c|c|c|c|c|c|}
\cline { 2 - 6 } \multicolumn{1}{l|}{} & Muy bajo & Bajo & $\begin{array}{c}\text { Término } \\
\text { medio }\end{array}$ & Alto & Muy alto & Media \\
\hline Conoces el Plan de Desarrollo & $6,55 \%$ & $11,06 \%$ & $24,15 \%$ & $34,76 \%$ & $23,48 \%$ & 3,58 \\
\hline $\begin{array}{l}\text { La oferta formativa es adecuada para tu } \\
\text { desarrollo }\end{array}$ & $5,34 \%$ & $10,67 \%$ & $25,06 \%$ & $36,43 \%$ & $22,51 \%$ & 3,60 \\
\hline $\begin{array}{l}\text { Las modalidades de aprendizaje son } \\
\text { adecuadas para tu desarrollo }\end{array}$ & $5,65 \%$ & $10,12 \%$ & $25,65 \%$ & $40,24 \%$ & $18,35 \%$ & 3,56 \\
\hline $\begin{array}{l}\text { Lo que has aprendido ha contribuido a } \\
\text { mejorar tu práctica docente }\end{array}$ & $4,90 \%$ & $10,26 \%$ & $24,71 \%$ & $38,23 \%$ & $21,91 \%$ & 3,62 \\
\hline $\begin{array}{l}\text { Nivel de satisfacción con el Plan de } \\
\text { Desarrollo }\end{array}$ & $7,75 \%$ & $11,03 \%$ & $30,28 \%$ & $38,26 \%$ & $12,68 \%$ & 3,37 \\
\hline
\end{tabular}


Con respecto al nivel de satisfacción con el PDD se ha estudiado la relación que tiene con las otras variables para demostrar la importancia de una planificación adecuada que llegue a los docentes. Para ello, se han calculado diversas medidas de asociación que se presentan en las siguientes líneas. En concreto, dado el carácter ordinal de las variables, las medidas utilizadas han sido: direccionales (d de Somer) y simétricas (Gamma, Tau-b de Kendall, Tau-c de Kendall y correlaciones Spearman). La hipótesis de contraste concierne a la existencia de relación estadísticamente significativa entre las valoraciones dadas por los docentes sobre el PDD y el nivel de satisfacción general respecto a éste. De esta forma se podrá demostrar la importancia que tiene planificar el PDD atendiendo a un modelo 70:20:10.

\section{Medidas direccionales}

Los resultados correspondientes a las medidas direccionales pueden (tabla 2). Atendiendo al estadístico d de Somer $(p=0,00)$ la probabilidad que se alcanza es inferior a $\alpha=0,05$ en todas las variables contrastadas. Este resultado nos permite rechazar la hipótesis de independencia y afirmar que las valoraciones dadas por los docentes sobre el PDD están relacionadas con el nivel de satisfacción que tienen con el mismo. También se observan medias positivas que indican una relación positiva entre el conocimiento que tienen los docentes sobre el Plan y su nivel de satisfacción. Por otro lado, el nivel de satisfacción con el PDD mejora, del mismo modo, cuando las oportunidades y modalidades de aprendizaje se consideran adecuadas, y cuando los docentes consideran que lo aprendido contribuye a mejorar la práctica docente.

Tabla 2: Medidas direccionales

\begin{tabular}{|c|c|c|c|c|}
\hline Medidas direccionales ( $d$ de Somers) & Valor & Error típ. asint. & T aproximada & Sig. aproximada \\
\hline Simétrica & 0,38 & 0,04 & 9,22 & 0,00 \\
\hline $\begin{array}{l}\text { Nivel de satisfacción con el Plan de Desarrollo- } \\
\text { dependiente }\end{array}$ & 0,38 & 0,04 & 9,22 & 0,00 \\
\hline Conoces el Plan de Desarrollo- dependiente & 0,38 & 0,04 & 9,22 & 0,00 \\
\hline Simétrica & 0,70 & 0,03 & 22,28 & 0,00 \\
\hline $\begin{array}{l}\text { Nivel de satisfacción con el Plan de Desarrollo- } \\
\text { dependiente }\end{array}$ & 0,70 & 0,03 & 22,28 & 0,00 \\
\hline $\begin{array}{l}\text { La oferta formativa es adecuada para tu } \\
\text { desarrollo- dependiente }\end{array}$ & 0,71 & 0,03 & 22,28 & 0,00 \\
\hline Simétrica & 0,75 & 0,02 & 25,90 & 0,00 \\
\hline $\begin{array}{l}\text { Nivel de satisfacción con el Plan de Desarrollo- } \\
\text { dependiente }\end{array}$ & 0,74 & 0,02 & 25,90 & 0,00 \\
\hline $\begin{array}{l}\text { Las modalidades de aprendizaje zona } \\
\text { adecuadas para tu desarrollo- dependiente }\end{array}$ & 0,75 & 0,02 & 25,90 & 0,00 \\
\hline Simétrica & 0,68 & 0,03 & 21,09 & 0,00 \\
\hline $\begin{array}{l}\text { Nivel de satisfacción con el Plan de Desarrollo- } \\
\text { dependiente }\end{array}$ & 0,68 & 0,03 & 21,09 & 0,00 \\
\hline $\begin{array}{l}\text { Lo que he aprendido ha contribuido a mejorar } \\
\text { mi práctica docente dependiente }\end{array}$ & 0,68 & 0,03 & 21,09 & 0,00 \\
\hline
\end{tabular}

\section{Medidas simétricas}

Las medidas simétricas de concordancia (tabla 3) muestran los mismos resultados que las medidas direccionales. En todos los estadísticos utilizados (Correlaciones, Gamma, Tau-b de Kendall y Tau-c de Kendall), la probabilidad asociada a los diferentes estadísticos $(p=0,00)$ siempre es inferior a $\alpha=0,05$, por lo que de nuevo se rechaza la hipótesis nula de independencia. Las medias en todos los estadísticos de contraste son positivas y nos permiten recalcar la existencia de una asociación moderada y positiva entre las variables estudiadas.

Tabla 3: Medidas simétricas

\begin{tabular}{|l|l|c|c|c|c|}
\hline \multicolumn{2}{|c|}{ Nivel de satisfacción con el Plan de Desarrollo } & Valor & $\begin{array}{c}\text { Error } \\
\text { típ.asint. }\end{array}$ & $\begin{array}{c}T \\
\text { aproximada }\end{array}$ & $\begin{array}{c}\text { Sig. } \\
\text { Aproximada }\end{array}$ \\
\hline \multirow{4}{*}{ Conoces el Plan de Desarrollo } & Tau-b de Kendall & 0,38 & 0,04 & 9,22 & 0,00 \\
& Tau-c de Kendall & 0,35 & 0,04 & 9,22 & 0,00 \\
& Gamma & 0,50 & 0,05 & 9,22 & 0,00 \\
& Correlación de Spearman & 0,43 & 0,05 & 9,74 & 0,00 \\
\hline \multirow{3}{*}{$\begin{array}{l}\text { La oferta formativa es adecuada } \\
\text { para tu desarrollo }\end{array}$} & Tau-b de Kendall & 0,70 & 0,03 & 22,28 & 0,00 \\
& Tau-c de Kendall & 0,64 & 0,03 & 22,28 & 0,00 \\
& Gamma & 0,86 & 0,03 & 22,28 & 0,00 \\
\hline
\end{tabular}


Tabla 3 (continuación)

\begin{tabular}{|l|l|c|c|c|c|}
\hline \multicolumn{2}{|c|}{ Nivel de satisfacción con el Plan de Desarrollo } & Valor & $\begin{array}{c}\text { Error } \\
\text { típ.asint. }\end{array}$ & $\begin{array}{c}T \\
\text { aproximada }\end{array}$ & $\begin{array}{c}\text { Sig. } \\
\text { Aproximada }\end{array}$ \\
\hline \multirow{2}{*}{$\begin{array}{l}\text { Las modalidades de aprendizaje } \\
\text { zona adecuadas para tu } \\
\text { desarrollo }\end{array}$} & Tau-b de Kendall & 0,75 & 0,02 & 25,90 & 0,00 \\
& Tau-c de Kendall & 0,68 & 0,03 & 25,90 & 0,00 \\
\hline \multirow{3}{*}{$\begin{array}{l}\text { Lo que he aprendido ha } \\
\text { contribuido a mejorar mi práctica } \\
\text { docente }\end{array}$} & Gamma & 0,91 & 0,02 & 25,90 & 0,00 \\
& Correlación de Spearman & 0,81 & 0,03 & 28,01 & 0,00 \\
\hline & Tau-b de Kendall & 0,68 & 0,03 & 21,09 & 0,00 \\
& Gau-c de Kendall & 0,62 & 0,03 & 21,09 & 0,00 \\
& Gamma & 0,84 & 0,03 & 21,09 & 0,00 \\
\hline
\end{tabular}

\section{Resultados cualitativos}

Del análisis cualitativo llevado a cabo en el grupo focal se desatacan tres resultados positivos. El primero de ellos tiene que ver con la posibilidad de personalizar el PDD de cada docente, consensuándolo con su responsable, en este caso con su director de departamento. El segundo aspecto está relacionado con la flexibilidad del profesor para realizar diferentes tipos de actividades formativas para su desarrollo. Y, por último, el tercer resultado se centra en la adquisición de una mayor autonomía para definir su desarrollo profesional. Según la percepción del claustro, el principal aspecto de mejora, coincidiendo con el estudio de lón y Cano (2012), es la excesiva carga de docencia y de gestión, ya que complica su dedicación al PDD. Un segundo aspecto es la demanda de un mayor acompañamiento y seguimiento del Plan por parte de su responsable, resultado también reflejado en las conclusiones del estudio realizado por Development Dimensions International (2015), en el que la falta de retroalimentación por parte del responsable es la principal barrera para adoptar el modelo aprendizaje basado en el trabajo. En tercer lugar, los profesores demandan más oportunidades de formación específica (técnica), siendo necesario ajustar mejor la oferta formativa a las necesidades de cada facultad y escuela.

\section{DISCUSIÓN Y ANALISIS FINAL}

A luz de los resultados de este estudio, en líneas generales, los aspectos con los que los profesores se muestran más satisfechos son el carácter flexible y personalizado del Plan. Además, las percepciones que tienen los docentes sobre el conocimiento del PDD, la oferta formativa, las modalidades de aprendizaje, y la contribución del aprendizaje en la mejora de la práctica docente son altas, al igual que su nivel de satisfacción. En cuanto a las modalidades de aprendizaje, la mayor tasa de participación sigue centrándose en la modalidad Descubrir, aunque se observa que la modalidad Experimentar comienza a despertar interés en el claustro. Estos resultados siguen la tendencia expuesta por Garavan et al. (2012), observándose una distribución muy distinta a la propuesta en el modelo teórico 70:20:10, pues más del 70\% de las actividades formativas corresponden a los cursos tradicionales (10\%). Es necesario, por tanto, seguir identificando formas de promover y reconocer el aprendizaje en el trabajo (Steinert et al. 2016).

Los resultados del análisis cuantitativo señalan que hay una relación positiva entre las cuatro variables analizadas (el conocimiento del PDD, la adecuación de la oferta formativa y de las modalidades de aprendizaje, y la contribución a la mejora de la práctica docente) y el nivel de satisfacción de los profesores con el Plan. Por último, algunas de las principales recomendaciones que han surgido como resultado de la evaluación instan a continuar trabajando en la consolidación del PDD. Así, es necesario reforzar, entre otros aspectos, los siguientes: impulsar el cambio cultural que este nuevo modelo de desarrollo de profesorado conlleva en la institución; dar mayor peso al aprendizaje colaborativo y a la experimentación como vías prioritarias de desarrollo; y actualizar y mejorar las actividades de aprendizaje del Plan. Por último, es necesario seguir llevando a cabo la evaluación continua del mismo y la medición de su eficacia como herramienta para el desarrollo docente.

\section{CONCLUSIONES}

El estudio realizado nos lleva a concluir que el conocimiento del PDD, de la oferta formativa, de las modalidades de aprendizaje y de la contribución del aprendizaje en la mejora de la práctica docente que tienen los profesores, se constituye como indicadores significativos del nivel de satisfacción con el PDD. Por lo tanto, una buena comunicación e información sobre el PDD, unido a la correcta planificación de las oportunidades y modalidades de aprendizaje, influye de manera decisiva en su satisfacción con el Plan. El diseño del PDD, basado en el modelo 70:20:10, ha tenido una gran aceptación dentro del claustro de profesores de la UEM. Todo ello nos insta a seguir avanzando en la consolidación y mejora del PDD para el desarrollo y la formación de nuestros docentes. 


\section{REFERENCIAS}

Boud, D. y A. Drew, Reconceptualising academic work as professional practice: implications for academic development, International Journal for Academic Development, 18(3), 208-221 (2013)

Bryman, A., Integrating quantitative and qualitative research: how is it done? Qualitative research, 6(1), 97-113 (2006)

Casal, J. y E. Mateu, Tipos de muestreo, Revista de Epidemiología y Medicina Preventiva, 1, 3-7 (2003)

Cross, J., Informal Learning: Rediscovering the Natural Pathways That Inspire Innovation and Performance, John Wiley \& Sons, San Fancisco, USA (2007)

De Rijdt, C., A. Stes, C. Vleuten y F. Dochy, Influencing variables and moderators of transfer of learning to the workplace within the area of staff development in higher education: Research Review, doi: 10.1016/j.edurev.2012.05.007, Educational Research Review, 8, 48-74 (2013)

Desimone, L. M., Improving impact studies of teachers' professional development: Toward better conceptualizations and measures, Educational Researcher, 38(3), 181-199 (2009)

Garavan, T.N., R. Carbery y A. Rock, Mapping talent development: definition, scope and architecture, European Journal of Training and Development, 36(1), 5-24 (2012)

Garmendia, M., J.I. Barragués, K. Zuza y J. Guisasola, Proyecto de formación del profesorado universitario de Ciencias, Matemáticas y Tecnología, en las metodologías de Aprendizaje Basado en Problemas y Proyectos, doi: 10.5565/rev/ensciencias.911, Enseñanza de las Ciencias, 32(2), 113-129 (2014)

Global leadership Forecast 2014-2015. Ready Now Leaders, Development Dimensions International (2015)

Ion, G. y E. Cano, La formación del profesorado universitario para la implementación de la evaluación por competencias, Educación XX1, 15(2), 249-270 (2012)

Jennings, C., L. Overton y G. Dixon, In-Focus Report: 70+20+10=100, The Evidence behind the Numbers, Towards Maturity (2016)

Krueger, R. A. y M.A. Casey, Focus groups: a practical guide for applied research, 4를 Ed., 1-15, Sage, Thousand Oaks, USA (2009)

Lombardo, M.M. y R.W. Eichinger, The Career Architect Development Planner, 4ª Ed., 4-13, Lominger, Minneapolis, USA (1996)

Madinabeitia, A. y C. Lobato, ¿Puede el impacto de las estrategias de desarrollo docente de larga duración cambiar la cultura institucional y organizativa en educación superior?, Educar, 51(1), 127-147 (2015)

Martínez, A.E., A.M. Cruz y otros tres autores, La observación formativa como instrumento de desarrollo en profesores universitarios en la Universidad Europea de Madrid, Revista Complutense de Educación, 29 (4), 1365-1380 (2018)

Mas, O. y P. Olmos, El profesor universitario en el espacio europeo de educación superior: La autopercepción de sus competencias docentes actuales y orientaciones para su formación pedagógica, Revista Mexicana de Investigación Educativa, 21(69), 437-470 (2016)

McCall, M. W. y C.D. McCauley, Using Experience to Develop Leadership Talent, 1-15, Jossey-Bas, San Francisco, USA (2014)

Oliver, D.H., A.H. Church, R. Lewis y E.I. Desrosiers, An integrated framework for assessing, coaching and developing global leaders, Advances in Global Leadership, 5, 195-224 (2009)

Princeton University, Learning process (2015)

Santos, C. y C. Sarceda, Desarrollo de Competencias Docentes en Educación Infantil: Una Experiencia Interdisciplinar en la Formación Inicial de Profesores, doi:10.4067/S0718-50062017000600005, Formación Universitaria, 10(6), 39-50 (2017)

Steinert, Y., K. Mann y otros diez autores, A systematic review of faculty development initiatives designed to enhance teaching effectiveness: A 10-year update: BEME Guide No. 40. Medical Teacher, 38(8), 769-786 (2016)

Tejada, J., Professionalisation of teaching in universities: Implications from a training perspective, RUSC Universities and Knowledge Society Journal, 10(1), 345-358 (2013)

Universidad Europea de Madrid, Modelo Académico (2019)

Vallejo, B. y U. Wehn, Capacity Development Evaluation: The Challenge of the Results Agenda and Measuring Return on Investment in the Global South, World Development, 79, 1-13 (2015)

Villarroel, V. A. y D.V. Bruna, Competencias Pedagógicas que caracterizan a un docente universitario de excelencia: Un estudio de caso que incorpora la perspectiva de docentes y estudiantes, doi:10.4067/S0718-50062017000400008, Formación Universitaria, 10(4), 75-96 (2017)

Webster-Wright, A., Reframing professional development through understanding authentic professional learning, Review of Educational Research, 79(2), 702-739 (2009)

Zabalza, M.A., Ser profesor Universitario hoy, La Cuestión Universitaria, (5), 68-80 (2016) 